\title{
Dimensionale Messtechnik in der digitalen Produktion - Aufgaben, Vernetzung und Schnittstellen
}

\author{
Dietrich Imkamp ${ }^{1}$ \\ ${ }^{1}$ Carl Zeiss Industrielle Messtechnik GmbH, Carl-Zeiss-Straße 22, D-73447 Oberkochen
}

\section{Zusammenfassung}

Digitalisierung ist heute ein prägender Begriff auch in der industriellen Produktion. Daraus hat sich der Begriff der „Digitalen Produktion“ entwickelt, Darunter wird einer Produktionsweise verstanden, in der die Informationen durchweg in digitaler Form erzeugt, verwaltet, gespeichert, verteilt, verarbeitet und kommuniziert werden [1]. Für die dimensionale Messtechnik, als der "Informationslieferant“" zur Qualität der Produktion, hat dieses Thema eine besondere Bedeutung. Die digitale Verarbeitung von Messergebnissen ist bereits weit verbreitet. Allerdings beschränkt sich die digitale Weiterverarbeitung dieser Informationen außerhalb der Messtechnik auf einzelne Anwendungsfelder. Die Übernahme von digitalen Informationen in die Messtechnik hat sich dagegen in den letzten Jahren erheblich weiterentwickelt. Darüber hinaus werden sich Geräte für dimensionale Messaufgaben mit allen relevanten Daten wie Werkzeugmaschinen in die digital gesteuerte Produktion integrieren.

Keywords: Dimensionale Messtechnik, Digitalisierung, Industrie 4.0

\section{Einführung}

Die Messtechnik wird in der Produktion vorwiegend zur Prüfung der hergestellten Produkte eingesetzt, wobei die Prüfung von geometrischen Maßen und deren Toleranzen [2] dominiert. Sie ist dazu informationstechnisch mit vielen anderen Bereichen des industriellen Produktionsprozesses verknüpft. An der Aufgabenstellung ändert sich in der digitalen Produktion nichts. Aber durch die Art der Verknüpfung mit digitalen Schnittstellen eröffnen sich neue Möglichkeiten der Kommunikation.

Der Schwerpunkt dieses Beitrags liegt bei der Koordinatenmesstechnik. Dabei wird die Produktoberfläche mit einem Messsystem punktweise erfasst, und die bezüglich eines geeigneten Koordinatensystems aufgenommen Punkte mit Hilfe von Verfahren der Ausgleichsrechnung zu Parametern der Produktgestalt (z. B.: Abstände, Parameter einer Flächenbeschreibung) verdichtet [2]. Sowohl zur Punkterfassung als auch zur anschließenden Auswertung werden Digitalrechner verwendet, so dass sich eine digitale Integration dieser Geräte in die Produktion anbietet.

Trotzdem ist zu ergänzen, dass heute auch andere dimensionale Messgeräte in der Produktion mit digitalen Schnittstellen ausgerüstet werden, um besonders
Messergebnisse rechnergestützt zu übertragen, so dass auf Papiermessprotokolle verzichtet werden kann. Diese Schnittstellen sind heute auch bei vielen einfachen Handmessmittel (z. B. Messschieber) verfügbar. Eine Integration dieser Messgeräte ist wesentlich einfacher als die Integration der komplexen Messsysteme in der Koordinatenmesstechnik.

\section{Der Prozess der Koordinatenmesstechnik und seine digitalen Schnittstellen}

Der Prozess der Koordinatenmesstechnik besteht aus drei Schritten: Messablauferzeugung, Messung und Auswertung [2] (Abbildung 1).

Bei der Messablauferzeugung wird festgelegt, welche Punkte zur Messung der Produktmerkmale erfasst werden. Bei einem Koordinatenmessgerät, mit dem ein einen Sensor bewegendes System bezeichnet wird [3], werden bei der Ablauferzeugung die Sensor-Verfahrbewegungen definiert. Für ein optisches Koordinatenmesssystem werden Ansichten bestimmt, um die erforderlichen Punkte zu erfassen [4]. Als Basis dient dazu in der digitalen Produktion ein zur Messsoftware übertragenes CAD-Modell des Produktes, das heute auch die Produktmerkmale enthält (Abbildung 2). Zur Übertragung der Produktmerkmale dienen mit dem geometrischen Produktmodell verknüpfe 
Attribute, die als „Product Manufacturing Information" (PMI) bezeichnet werden [5].

Für Koordinatenmessgeräte existiert zur digitalen Übertragung von Messabläufen das DMIS-Format. Sein funktionaler Umfang deckt jedoch nur Punkt- und Liniensensoren ab [6]. Herstellerspezifische Erweiterungen zur Vergrößerung des Funktionsumfangs schränken die Übertragungsmöglichkeiten an unterschiedliche Geräte stark ein.

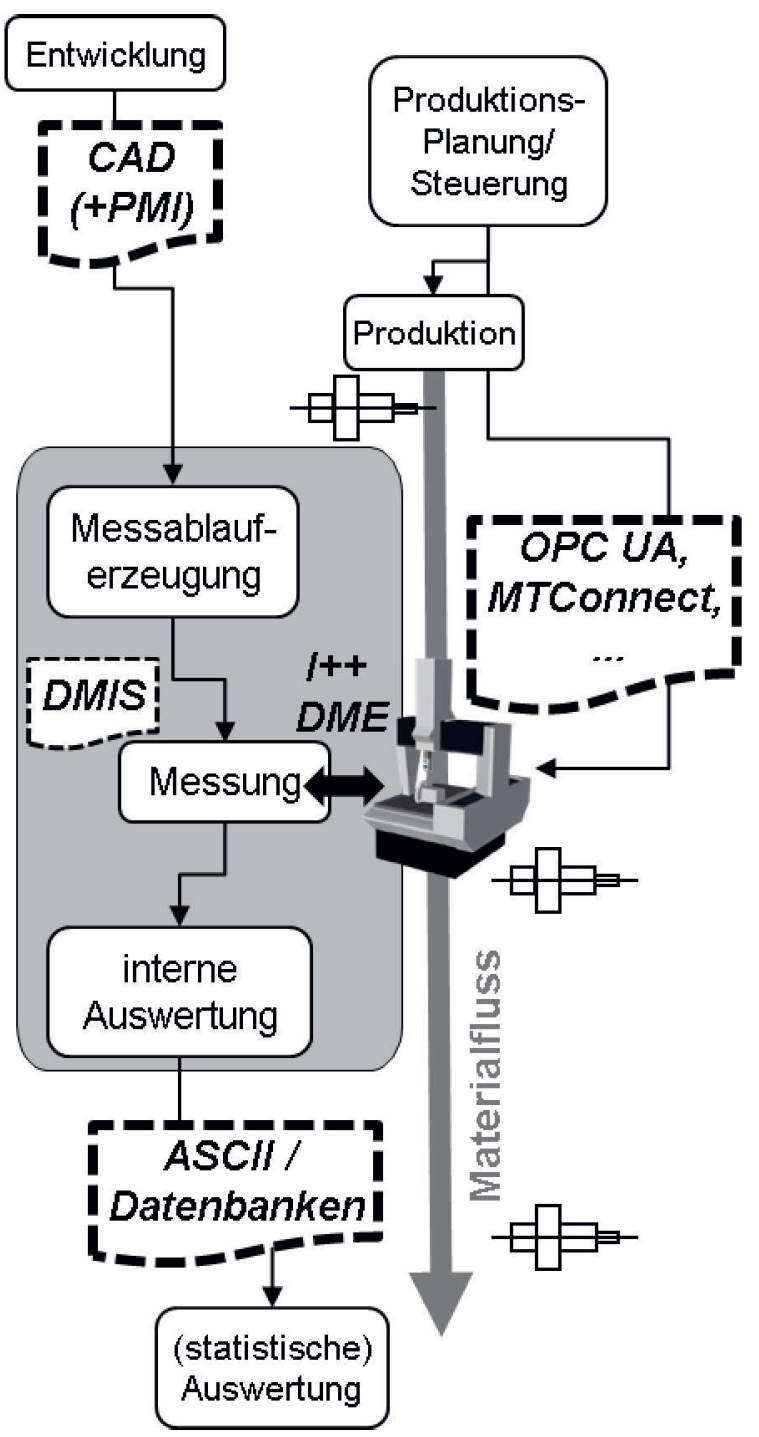

Abb. 1: Prozess der Koordinatenmesstechnik mit digitalen Schnittstellen zur Integration in die Produktion

Die Messung wird durch ein Koordinatenmesssystem ausgeführt [2]. Im Bereich der Koordinatenmessgeräte gibt es zur Kommunikation zwischen Gerätesteuerung und Messsoftware das standardisierte Format „I++ DME“ [7]. Es allerdings genauso wie DMIS funktional auf bestimmte Sensoren beschränkt.
Die Ergebnisse der Koordinatenmessung werden nach der internen Auswertung (Verdichtung der aufgenommenen Koordinatenpunkte zu Parametern der Produktgestalt, Filterung der Punkte, usw. [2]) vorwiegend in verschiedenen ASCII-Formaten (z. B.: DMIS-Out, Q-DAS) oder über geeignete Softwareschnittstellen direkt an Datenbanken digital weitergegeben. Ein allgemein anwendbarer Standard hat sich an dieser Stelle nicht durchgesetzt [6].

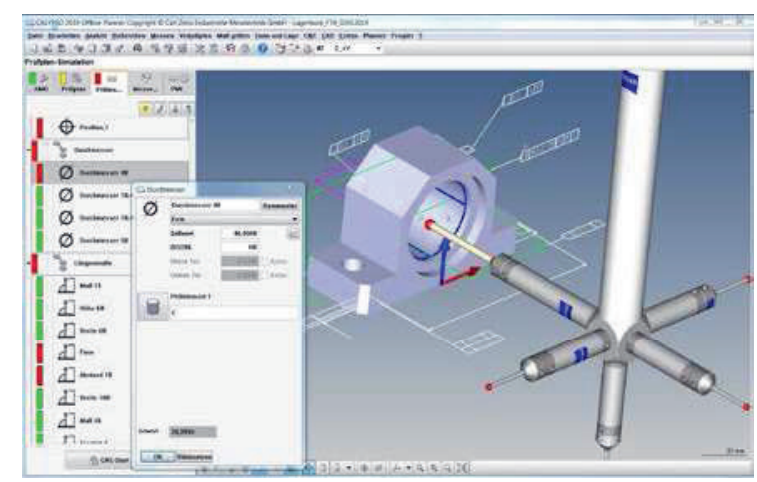

Abb. 2: CAD-Modell mit Maßen und Toleranzen in einer Software für Koordinatenmessgeräte mit Visualisierung des Tasters

Die Messergebnisse werden einerseits genutzt, um die Konformität mit den Produktanforderungen zu prüfen und andererseits, um in vorgelagerten Bereichen Verbesserung durchzuführen, so dass Regelkreise entstehen [2]. Dabei werden unterschiedliche Übertragungsformate und Formen der Ergebnisdarstellung benutzt. Bei einzelnen Produktionsverfahren existieren auch automatische Verfahren zur Rückführung auf Basis digitaler Informationen (z. B.: Voreinstellung von Erodier-Maschinen auf Basis von Koordinatenmessungen) [6].

Die Beschreibung des Prozesses der Koordinatenmesstechnik verdeutlicht die Komplexität der dazu erforderlichen Schnittstellen und Datenstrukturen [8]. Mit dem QIF (Quality Information Framework) Standard $[9,10]$ wird durch einen ganzheitlichen Ansatz versucht, diese Komplexität zu beherrschen. Der Standard besteht aus 6 Anwendungsmodellen, einer von allen Modellen genutzten Bibliothek sowie einer Verknüpfung zu dem hier zuvor bereits erwähnten DMIS Standard. Informationen zur Produktspezifikation einschließlich der Produktgestalt werden von einem CAD-Modell mit PMls (s.o.) bezogen. Als Datenformat wird XML benutzt. 


\section{Digitaler Schatten und Digitale Zwilling der Koordinatenmesstechnik}

Unter einem Digitalen Schatten wird in der Produktionstechnik ein Abbild der Prozesse in der Produktion, der Entwicklung und angrenzenden Bereichen verstanden mit dem Zweck, eine echtzeitfähige Auswertungsbasis aller relevanten Daten zu schaffen. Der Digitale Schatten überführt zunächst den realen Prozess in die virtuelle Welt. Der Digitale Zwilling kann darauf aufbauend durch ein Prozessmodell und Simulation ein möglichst identisches Abbild der Realität liefern [11].

Im Zusammenhang mit der Koordinatenmesstechnik spielen Modelle des Messprozesses zur Korrektur systematischer Abweichung schon seit ihren Anfängen eine wichtige Rolle [12]. Heute kommen darauf aufbauend Modelle zur Abschätzung der Messunsicherheit durch Simulation, die unter dem Begriff der „virtuellen Messgeräte" bekannt geworden sind, zur Anwendung [13].

Weiterhin werden bei der Off-Line Programmierung von Messabläufen digitale Zwillinge der Produkte mit ihren Aufspannvorrichtungen, die als CAD Modelle importiert werden, zusammen mit digitalen Zwillingen der Geräte zur Sensor-VerfahrwegFestlegung und Kollisionskontrolle durch Verfahrweg-Simulation genutzt (Abbildung 3).

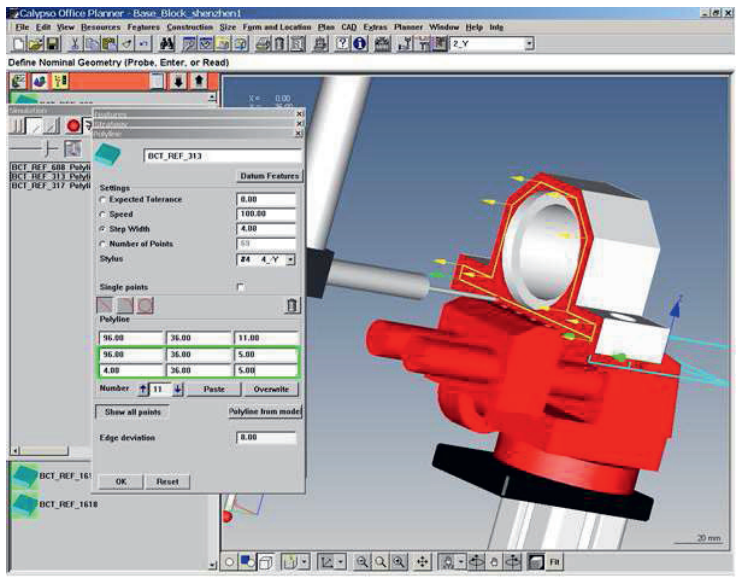

Abb. 3: Kollisionskontrolle durch VerfahrwegSimulation an einem Digitalen Zwilling des Produktes mit Aufspannvorichtung

Diese digitalen Zwillinge ermöglichen eine effizientere Nutzung der Koordinatenmesstechnik durch die Reduzierung und Bestimmung der Messunsicherheit sowie die Off-Line Programmierung zur Messablauferstellung ohne Messgerät. Zur Integration der Koordinatenmesstechnik in ein digitales Abbild der Produktion dienen diese Zwillinge aber nicht.

\section{Digitale Integration der Koordinatenmesstechnik}

Für die Integration der Koordinatenmesstechnik in das digitale Abbild der Produktion und damit auch in Produktionsplanung und Steuerung (Abbildung 1) ist ein digitaler Schatten der real verfügbaren Koordinatenmesssysteme entsprechend der zuvor beschriebenen Abgrenzung zum digitalen Zwilling erforderlich. Dazu sind alle relevanten Daten eines Koordinatenmesssystems zu definieren, und sie über eine geeignete Schnittstelle digital zur Verfügung zu stellen. Als Schnittstelle bieten sich dazu die neuen Entwicklungen im Bereich der Produktionstechnik wie MTConnect [14] aus den USA oder OPC UA (Open Platform Communications Unified Architecture) [15] aus Europa an. Beide Standards bieten unterschiedliche Modelle zur Beschreibung von Systemen in der Produktion an. Für Koordinatenmesssysteme gibt es bis auf eine prototypenhafte Implementierung für MTConnect mit beschränktem Funktionsumfang keine Vorlage [16]. Daher wird hier ein hierarchisches Datenmodell für ein Koordinatenmesssystem aufgestellt und in Form eines einfachen Entity-Relationship Diagramms dargestellt (Abbildung 4).

Das Modell lehnt sich an die MTConnect Beschreibung eines "Devices“ an. Es beschreibt ein Koordinatenmesssystem als Gerät (Device) mit seinen Eigenschaften und untergeordneten Komponenten (Components). Diese Daten bilden die weitgehend unveränderlichen Stammdaten des Systems.

Zusätzlich werden der aktuelle Gerätezustand einschließlich Informationen über die Umgebungsbedingungen und die Fähigkeit bestimmte Messabläufe unter Berücksichtigung der Anforderungen an die Messunsicherheit auszuführen dargestellt. Über die ausführbaren Messabläufe wird eine Verbindung zu den Produkten und ihren Produktmerkmalen hergestellt. Diese Daten verändern sich während des Geräteeinsatzes und werden laufend aktualisiert. Diese Aktualisierung erfolgt automatisch beispielsweise bei den Umgebungsbedingungen, die durch Sensoren erfasst werden, oder durch manuelle Eingabe beispielsweise in Form der Bestätigung einer erfolgreich durchgeführten Kalibrierung.

Schließlich speichert das Gerät Daten zur Historie beispielsweise zu Kollisionen, um den 
Gerätezustand kontinuierlich zu dokumentieren. Diese Informationen können dazu dienen, die Richtigkeit von Messergebnissen aus der Vergangenheit zu bestätigen, oder vorausschauende Wartungen („Predictive Maintenance“) zu planen [17].

Die Kategorisierung der Daten des Systems in Stammdaten, Bewegungsdaten und Historiendaten orientiert sich an den Datenstrukturen, die schon heute typischerweise in der Prüfmittelüberwachung verwendet werden [2]. Mit der automatisierten digitalen Bereitstellung dieser Daten lässt sich die Überwachungsdichte einerseits verdichten und andererseits effizienter gestalten.

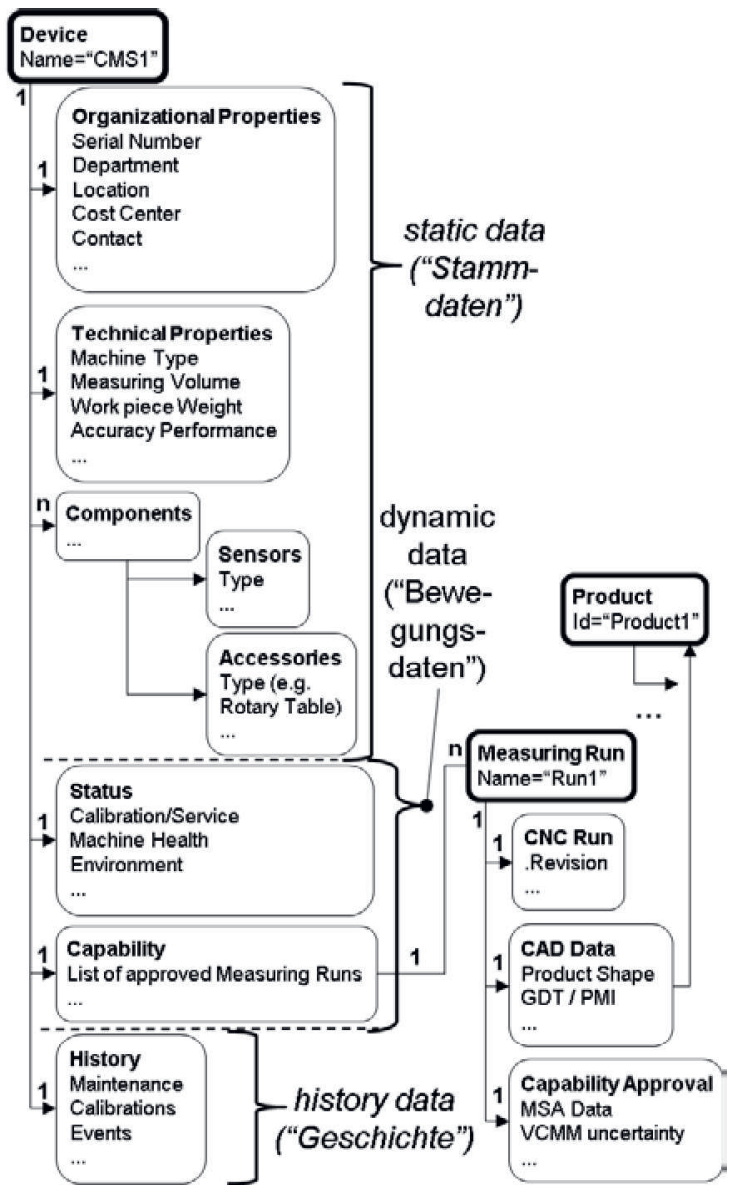

\section{Abb. 4: Datenstruktur für einen digitalen Schatten eines Koordinatenmesssystems}

Die Daten dieses digitalen Schattens können durch die Produktion genutzt werden, um ein geeignetes Messgerät zur Prüfung eines bestimmten Produktes zu finden. Das Prüfmittelmanagement kann auf Basis der Daten Wartungen und Kalibrierungen planen. Dieser Ansatz eines Datenmodells für einen digitalen Schatten eines Koordinatenmesssystems ist nicht vollständig, und die
Anwendungsszenarien für die Daten werden Ergänzungen und zusätzliche Verknüpfungen erfordern. Dennoch machen die Anwendungsbeispiele deutlich, dass ein Koordinatenmesssystem wie eine Werkzeugmaschine in die digitale Produktion über die gleichen Schnittstellen integrierbar ist.

\section{Zusammenfassung}

Der digitale Informationsfluss für ein Koordinatenmesssystem und die mögliche Darstellung seiner Datenstruktur für einen digitalen Schatten zeigen die Möglichkeiten der Einbindung von Koordinatenmesssystemen in die digitale Produktion auf.

\section{Literaturnachweis}

[1] Westkämper, E. Spath, D., Constantinescu, C., Lentes, J.: Digitale Produktion, Verlag: Springer, 2013.

[2] Pfeifer, T., Schmitt, R.: Fertigungsmesstechnik, Oldenbourg Verlag, München 2010.

[3] DIN EN ISO 10360-1 Geometrische Produktspezifikation (GPS), Annahmeprüfung und Bestätigungsprüfung für Koordinatenmessgeräte (KMG), Teil 1: Begriffe Juli 2003.

[4] Imkamp, D., Wanner, J.: Vom Messablaufplan über die Programmierung, Durchführung und Auswertung bis zur Ergebnis-darstellung, in: Weckenmann, A. (Hrsg.): Koordinatenmesstechnik: Flexible Strategien für funktions- und fertigungsgerechtes Prüfen. Carl Hanser Verlag, München 2012.

[5] Imkamp, D., Gabbia, A.: Nutzung des PMI Standards zur automatisierten Übertragung von Merkmalsdaten in die dimensionale Messtechnik, in: ITG-Fachbericht 250, Sensoren und Messsysteme 2014 zur 17. ITG/GMA-Fachtagung Sensoren und Messsysteme 2014 vom 03. bis 04. Juni 2014 in Nürnberg.

[6] Pfeifer, T., Imkamp, D.: Koordinatenmesstechnik und CAx-Anwendungen in der Produktion Grundlagen, Schnittstellen, Integration, Carl Hanser Verlag, München 2004.

[7] Imkamp, D., Resch, J., Puntigam, W.: Nutzung der neuen hersteller-neutralen I++ DME Schnittstelle bei der Prüfung der Messabweichungsangaben von Koordinatenmessgeräten, in: Vorträge der 13. ITG/GMA-Fachtagung, Sensoren und Messsysteme in Freiburg/Breisgau, Germany, 13./14. März 2006, VDE Verlag GmbH, Berlin 2006.

[8] Evans, J., Frechette, S., Horst, J., Huang, H., Kramer, T., Messina, E., Proctor, F., Rippey, B., Scott, H., Vorburger, T., Wavering, A.: Analysis of Dimensional Metrology Standards (NISTIR 6847), National Institute of Standards and Technology (NIST), USA 2001

[9] ANSI/DMSC QIF 3.0 Quality Information Framework. Digital Metrology Standards Consortium, Inc. (DMSC), USA, 2018. 
[10] Zhao, Y. F., Horst, J. A., Kramer, T. R., Rippey, W., Brown, R. J.: Quality Information

Framework - Integrating Metrology Processes, in: IFAC Proceedings Volumes 45, Issue 6, 2325 May 2012, Pages 1301-1308,

Internetadresse: https://doi.org/10.3182/20120523-3-RO2023.00113.

[11] Bauernhansl, T.; Krüger, J.; Reinhart, G. et al. (Hrsg.: Wissenschaftliche Gesellschaft für Produktionstechnik Wgp e.v.): Wgp-Standpunkt Industrie 4.0, Internetadresse: www.wgp.de/uploads/media/WGPStandpunkt_Industrie_4-0.pdf. Zuletzt aufgerufen am 25.04.2019.

[12] Wäldele, F.: Genauigkeitssteigerung von Koordinatenmessgeräten durch numerische Fehlerkorrektur. In: Neumann, H. J. (Hrsg.): Präzisionsmesstechnik in der Fertigung mit Koordinatenmessgeräten, Expert Verlag, Ehningen bei Böblingen 1988.

[13] Schmitt, R., et. al.: Virtuelle Messgeräte: Definition und Stand der Entwicklung, in: tm Technisches Messen, Oldenbourg Industrieverlag, 75 (2008) 5, S. 298-310.

[14] MTConnect Standard, Version 1.4.0 (ANSI/MTC1.4-2018). MTConnect Institute, AMT - The Association For Manufacturing Technology, USA (22.04.2019: https://www.mtconnect.org/standarddownload20181).

[15] IEC 62541 OPC Unified Architecture. Part 1-14, 100, 2010-2018.

[16] MTConnectToolbox: https://github.com/johnmichaloski/MTConnectT oolbox Zuletzt aufgerufen am 25.04.2019.

[17] Imkamp, D., Grieser C.: Onboard Diagnostics Ein innovatives System zur Maschinenüberwachung. In: Sensoren und Messsysteme 2004, Tagung Ludwigsburg 15.16.3.2004 (VDI-Berichte 1829), VDI-Verlag, Düsseldorf 2004, S.785-789. 